\title{
Quantum-Trajectory Approach to Time-Dependent Transport in Mesoscopic Systems with Electron-Electron Interactions
}

\author{
X. Oriols* \\ Departament d'Enginyeria Electrònica, Universitat Autònoma de Barcelona, 08193 Bellaterra, Spain
}

(Received 17 July 2006; published 7 February 2007)

\begin{abstract}
It is proved that many-particle Bohm trajectories can be computed from single-particle time-dependent Schrödinger equations. From this result, a practical algorithm for the computation of transport properties of many-electron systems with exchange and Coulomb correlations is derived. As a test, two-particle Bohm trajectories in a tunneling scenario are compared to exact results with an excellent agreement. The algorithm opens the path for implementing a many-particle quantum transport (Monte Carlo) simulator, beyond the Fermi liquid paradigm.
\end{abstract}

DOI: 10.1103/PhysRevLett.98.066803

From a computational point of view, the direct solution of the many-particle Schrödinger equation is inaccessible for more than very few electrons. This issue is at the heart of almost all the unsolved problems in quantum transport. The standard solution to overcome this computational barrier is assuming noninteracting (Fermi liquid) electrons and decoupling the studies of transport from those of the electronic structure (via the effective electron mass) [1]. Nowadays, $a b$ initio many-particle quantum transport approaches, based on the density functional theory (DFT) [2], are being developed [3] to surpass the previous approximations.

In this Letter, we present an alternative approach, beyond the Fermi liquid paradigm, to study many-particle quantum transport using Bohm trajectories $[4,5]$. Bohmian mechanics was originally presented as an interpretative tool, and it generated an intense debate about the "reality" of the trajectories [6]. Here, this issue becomes irrelevant because Bohm trajectories (similarly to Feynman paths) are used to reproduce the probabilistic results of standard quantum mechanics. Following this point of view, Bohmian mechanics has recently undergone a revival to develop new quantum computational algorithms [7].

We study a system of $\mathbf{N}$ (spinless) electrons described by a (first-quantization) many-particle wave-function, $\boldsymbol{\Phi}(\vec{x}, t)$, solution of the Schrödinger equation:

$$
i \hbar \frac{\partial \boldsymbol{\Phi}(\vec{x}, t)}{\partial t}=\left\{\sum_{a=1}^{N}-\frac{\hbar^{2}}{2 m} \frac{\partial^{2}}{\partial x_{a}^{2}}+U(\vec{x}, t)\right\} \boldsymbol{\Phi}(\vec{x}, t),
$$

where $\vec{x} \equiv\left\{x_{1}, x_{2}, \ldots, x_{N}\right\} \equiv\left\{x_{a}, \vec{x}_{b}\right\}$ is the vector of the electron positions. For simplicity, we consider a 1D solidstate system where the lattice-electron interaction is included into the electron effective mass, $m$. The potential energy $U(\vec{x}, t)$ takes into account the Coulomb interaction among all electrons and the role of an external battery.

First, we summarize the basic many-particle de BroglieBohm development $[4,5,7,8]$. Equation (1) can be split into two (real) equations when the wave function is written in polar form, $\boldsymbol{\Phi}(\vec{x}, t)=R(\vec{x}, t) \exp [i S(\vec{x}, t) / \hbar]$. The real part leads to the many-particle quantum Hamilton-Jacobi
PACS numbers: 73.23. $-\mathrm{b}, 02.70 . \mathrm{Tt}, 34.10 .+\mathrm{x}$

equation:

$$
\frac{\partial S(\vec{x}, t)}{\partial t}+U(\vec{x}, t)+\sum_{a=1}^{N}\left\{K_{a}(\vec{x}, t)+Q_{a}(\vec{x}, t)\right\}=0,
$$

where the kinetic energy, $K_{a}(\vec{x}, t)$, and the so-called quantum potential energy, $Q_{a}(\vec{x}, t)$, associated with the $x_{a}$ electron are

$K_{a}(\vec{x}, t)=\frac{1}{2 m}\left(\frac{\partial S(\vec{x}, t)}{\partial x_{a}}\right)^{2}, \quad Q_{a}(\vec{x}, t)=-\frac{\hbar^{2}}{2 m} \frac{\partial^{2} R(\vec{x}, t) / \partial x_{a}^{2}}{R(\vec{x}, t)}$

Equations (2) and (3) provide a definition of the velocity $v_{a}(\vec{x}, t)$ for the electron labeled by the variable $x_{a}$. Therefore, a trajectory, $x_{a}[t]$, can be computed from

$$
\begin{aligned}
x_{a}[t] & =x_{a}\left[t_{o}\right]+\int_{t_{o}}^{t} v_{a}\left(\vec{x}\left[t^{\prime}\right], t^{\prime}\right) d t^{\prime}, \\
v_{a}(\vec{x}, t) & =\frac{1}{m} \frac{\partial S(\vec{x}, t)}{\partial x_{a}} .
\end{aligned}
$$

The vector $\vec{x}[t]=\left\{x_{1}[t], \ldots, x_{N}[t]\right\}=\left\{\vec{x}_{a}[t], \vec{x}_{b}[t]\right\}$ contains the $N$ Bohm trajectories. We omit the dependence of each trajectory on its particular initial position, $x_{a}\left[t_{o}\right]$. On the other hand, the imaginary part of Eq. (1) leads to a continuity equation:

$$
\frac{\partial R^{2}(\vec{x}, t)}{\partial t}+\sum_{a=1}^{N} \frac{\partial}{\partial x_{a}}\left[R^{2}(\vec{x}, t) v_{a}(\vec{x}, t)\right]=0 .
$$

Equation (5) guarantees that, at any time $t$, the observable results can be exactly computed from Bohm trajectories $[4,5,8]$. The only requirement is that the trajectories have to reproduce $R^{2}\left(\vec{x}, t_{o}\right)=\left|\boldsymbol{\Phi}\left(\vec{x}, t_{o}\right)\right|^{2}$ at one initial time, $t_{o}$, and move according to (4). Next, we demonstrate the main result of this Letter.

Theorem. - Any trajectory $x_{a}[t]$ that belongs to a particular set of $N$ Bohm trajectories $\vec{x}[t]=\left\{x_{a}[t], \vec{x}_{b}[t]\right\}$ as- 
sociated with a many-particle wave-function $\boldsymbol{\Phi}(\vec{x}, t)$ solution of Eq. (1) with $U(\vec{x}, t) \equiv U_{a}\left(x_{a}, \vec{x}_{b}, t\right)+U_{b}\left(\vec{x}_{b}, t\right)$ can be obtained from a single-particle wave function $\boldsymbol{\Psi}_{a}\left(x_{a}, t\right)$. This wave function is a solution of the following single-particle Schrödinger equation:

$$
i \hbar \frac{\partial \boldsymbol{\Psi}_{a}\left(x_{a}, t\right)}{\partial t}=\left\{-\frac{\hbar^{2}}{2 m} \frac{\partial^{2}}{\partial x_{a}^{2}}+U_{a}\left(x_{a}, \vec{x}_{b}[t], t\right)+G_{a}\left(x_{a}, \vec{x}_{b}[t], t\right)+i J_{a}\left(x_{a}, \vec{x}_{b}[t], t\right)\right\} \boldsymbol{\Psi}_{a}\left(x_{a}, t\right),
$$

where

$$
\begin{aligned}
& G_{a}\left(x_{a}, \vec{x}_{b}, t\right)=U_{b}\left(\vec{x}_{b}, t\right)+\sum_{k=1, k \neq a}^{N}\left\{K_{k}(\vec{x}, t)+Q_{k}(\vec{x}, t)-\frac{\partial S(\vec{x}, t)}{\partial x_{k}} v_{k}(\vec{x}[t], t)\right\}, \\
& J_{a}\left(x_{a}, \vec{x}_{b}, t\right)=\sum_{k=1, k \neq a}^{N} \frac{\hbar}{2 R^{2}(\vec{x}, t)}\left\{\frac{\partial R^{2}(\vec{x}, t)}{\partial x_{k}} v_{k}(\vec{x}[t], t)-\frac{\partial}{\partial x_{k}}\left(\frac{R^{2}(\vec{x}, t)}{m} \frac{\partial S(\vec{x}, t)}{\partial x_{k}}\right)\right\} .
\end{aligned}
$$

Proof.-First, we define the single-particle wave function, $\boldsymbol{\Psi}_{a}\left(x_{a}, t\right)=r_{a}\left(x_{a}, t\right) \exp \left[i s_{a}\left(x_{a}, t\right) / \hbar\right]$ from the manyparticle wave function $\boldsymbol{\Phi}(\vec{x}, t)$ evaluated at $\left\{x_{a}, \vec{x}_{b}[t]\right\}$. In particular, we define $r_{a}\left(x_{a}, t\right)=R\left(x_{a}, \vec{x}_{b}[t], t\right)$ and $s_{a}\left(x_{a}, t\right)=S\left(x_{a}, \vec{x}_{b}[t], t\right)$. From this definition, it is quite simple to realize that the same velocity (trajectory $x_{a}[t]$ ) is obtained either using $\boldsymbol{\Psi}_{a}\left(x_{a}, t\right)$ or $\boldsymbol{\Phi}(\vec{x}, t)$ in (4). Second, we show that $\boldsymbol{\Psi}_{a}\left(x_{a}, t\right)$ is generated by the following single-particle (complex) potential energy: $u_{a}\left(x_{a}, t\right)=$ $U_{a}\left(x_{a}, \vec{x}_{b}[t], t\right)+G_{a}\left(x_{a}, \vec{x}_{b}[t], t\right)+i J_{a}\left(x_{a}, \vec{x}_{b}[t], t\right)$. Ву rewriting a single-particle Schrödinger equation, the potential profile can be identified as $u_{a}\left(x_{a}, t\right) \equiv$ $\left[i \hbar \partial \boldsymbol{\Psi}_{a}\left(x_{a}, t\right) / \partial t+\left(\hbar^{2} / 2 m\right) \partial^{2} \boldsymbol{\Psi}_{a}\left(x_{a}, t\right) / \partial x_{a}^{2}\right] / \boldsymbol{\Psi}_{a}\left(x_{a}, t\right)$ when $\boldsymbol{\Psi}_{a}\left(x_{a}, t\right) \neq 0$ and $u_{a}\left(x_{a}, t\right) \equiv 0$ otherwise. The real part of $u_{a}\left(x_{a}, t\right)$, written in polar form, leads to $\operatorname{Re}\left[u_{a}\left(x_{a}, t\right)\right]=-\partial s_{a}\left(x_{a}, t\right) / \partial t-m v_{a}^{2}\left(x_{a}, \vec{x}_{b}[t], t\right) / 2+$ $\left(\hbar^{2} / 2 m\right)\left[\partial^{2} r_{a}\left(x_{a}, t\right) / \partial x_{a}^{2}\right] / r_{a}\left(x_{a}, t\right)$. When this result is rewritten in terms of $R\left(x_{a}, \vec{x}_{b}[t], t\right)$ and $S\left(x_{a}, \vec{x}_{b}[t], t\right)$, together with the Hamilton-Jacobi Eq. (2) evaluated at $\left\{x_{a}, \vec{x}_{b}[t]\right\}$, the terms $U_{a}\left(x_{a}, \vec{x}_{b}[t], t\right)+G_{a}\left(x_{a}, \vec{x}_{b}[t], t\right)$ are obtained. The imaginary part of $u_{a}\left(x_{a}, t\right)$ leads to $\operatorname{Im}\left[u_{a}\left(x_{a}, t\right)\right]=\hbar /\left[2 r_{a}^{2}\left(x_{a}, t\right)\right]\left\{\partial r_{a}^{2}\left(x_{a}, t\right) / \partial t+\partial\left[r_{a}^{2}\left(x_{a}, t\right) \times\right.\right.$ $\left.\left.v_{a}\left(x_{a}, \vec{x}_{b}[t], t\right)\right] / \partial x_{a}\right\}$. When this result is rewritten in terms of $R\left(x_{a}, \vec{x}_{b}[t], t\right)$ and $S\left(x_{a}, \vec{x}_{b}[t], t\right)$, together with the continuity Eq. (5), expression (6c) evaluated at $\left\{x_{a}, \vec{x}_{b}[t]\right\}$ is obtained. Q.E.D.

In the rest of the Letter, we explain two practical algorithms to compute $N$-particle Bohm trajectories without knowing the $N$-particle wave function, $\boldsymbol{\Phi}(\vec{x}, t)$, but using the previous theorem. Our algorithms have similarities with the original work of Kohn and Sham on the DFT [2]: the formidable simplification on the many-particle computations [9] comes at the price that some terms of the potential energy of the corresponding single-particle Schrödinger equations are unknown [the exchangecorrelation functional in the DFT and, here, the terms (6b) and (6c)].

First algorithm. -We consider a system of $N$ electrons with Coulomb interaction but without exchange interaction. As mentioned, the solution of Eq. (6a) needs educated guesses for the terms (6b) and (6c). Since no exchange interaction is considered, the correlation be- tween the $x_{a}$ electron and the rest is mainly contained in the term $U_{a}\left(x_{a}, \vec{x}_{b}[t], t\right)$. Thus, we can assume a zero-order Taylor expansion of the terms (6b) and (6c) in the variable $x_{a}$ around $x_{a}[t]$ to obtain $G_{a}\left(x_{a}, \vec{x}_{b}[t], t\right) \approx$ $G_{a}\left(x_{a}[t], \vec{x}_{b}[t], t\right)$ and $J_{a}\left(x_{a}, \vec{x}_{b}[t], t\right) \approx J_{a}\left(x_{a}[t], \vec{x}_{b}[t], t\right)$. Later, we will check this approximation. Then, the wave-function solution of (6a) can be written as $\boldsymbol{\Psi}_{a}\left(x_{a}, t\right) \approx \tilde{\boldsymbol{\Psi}}_{a}\left(x_{a}, t\right) \exp \left[\alpha_{a}(t) / \hbar-i \beta_{a}(t) / \hbar\right] \quad$ where $\alpha_{a}(t)=\int_{t_{o}}^{t} J_{a}\left(x_{a}\left[t^{\prime}\right], \vec{x}_{b}\left[t^{\prime}\right], t^{\prime}\right) d t^{\prime}+\alpha_{a}\left(t_{o}\right)$ and $\beta_{a}(t)=$ $\int_{t_{o}}^{t} G_{a}\left(x_{a}\left[t^{\prime}\right], \vec{x}_{b}\left[t^{\prime}\right], t^{\prime}\right) d t^{\prime}+\beta_{a}\left(t_{o}\right)$. The wave function $\tilde{\boldsymbol{\Psi}}_{a}\left(x_{a}, t\right)$ is the solution of Eq. (6a) with $u_{a}\left(x_{a}, t\right)=$ $U_{a}\left(x_{a}, \vec{x}_{b}[t], t\right)$. Since the velocity in (4) does not depend on the terms $\beta_{a}(t)$ and $\alpha_{a}(t)$, we do not have to compute them explicitly. We fix the initial many-particle wave function in Eq. (1) as a product of single-particle wave functions, $\boldsymbol{\Phi}\left(\vec{x}, t_{o}\right)=\chi_{1}\left(x_{1}, t_{o}\right) \cdots \chi_{N}\left(x_{N}, t_{o}\right)$. Then, the initial boundary condition for solving $\tilde{\mathbf{\Psi}}_{a}\left(x_{a}, t\right)$ is just $\tilde{\boldsymbol{\Psi}}_{a}\left(x_{a}, t_{o}\right)=\chi_{a}\left(x_{a}, t_{o}\right)$.

As a test, we consider a two-particle system $\vec{x}=\left\{x_{1}, x_{2}\right\}$ in a triple barrier $\mathrm{Al}_{x} \mathrm{Ga}_{1-x} \mathrm{As} / \mathrm{In}_{x} \mathrm{Ga}_{1-x} \mathrm{As}$ structure (which can be a rich mesoscopic scenario for resonant tunneling and Coulomb blockade) with $0.526 \mathrm{eV}$ barriers and a relative electron effective mass equal to 0.078 (see insets of Fig. 1). We control the strength of the Coulomb interaction by the lateral area, $A$, as described in Ref. [10]. The initial wave function, at $t_{o}=0$, is a product of two Gaussian wave packets, $\chi_{1}\left(x_{1}, t_{o}\right)$ and $\chi_{2}\left(x_{2}, t_{o}\right)$, whose central kinetic energies and positions are $0.180 \mathrm{eV}$ and $-62 \mathrm{~nm}$ for the first, and $0.225 \mathrm{eV}$ and $-75 \mathrm{~nm}$ for the second. The spatial dispersion is $25 \mathrm{~nm}$ for both. The exact two-particle Bohm trajectories $\vec{x}[t]=\left\{x_{1}[t], x_{2}[t]\right\}$ are computed using (4) after solving Eq. (1) numerically (see the 2D Bohm trajectories in Fig. 1). Alternatively, the Bohm trajectories are also computed by solving Eq. (6) with $u_{1}\left(x_{1}, t\right)=U_{1}\left(x_{1}, x_{2}[t], t\right)$ and $\tilde{\Psi}_{1}\left(x_{1}, t_{o}\right) \equiv \chi_{1}\left(x_{1}, t_{o}\right)$ for $x_{1}[t]$, and $u_{2}\left(x_{2}, t\right)=U_{2}\left(x_{1}[t], x_{2}, t\right)$ and $\tilde{\boldsymbol{\Psi}}_{2}\left(x_{2}, t_{o}\right) \equiv$ $\chi_{2}\left(x_{2}, t_{o}\right)$ for $x_{2}[t]$, simultaneously (see the 1D Bohm trajectories in Fig. 1). Figure 1 show the excellent agreement between exact Bohm trajectories and those computed within our algorithm [9]. In Fig. 1(a), the lateral area $A$ is so large that it makes the Coulomb interaction quite negligible [10]. The first electron is transmitted while the 


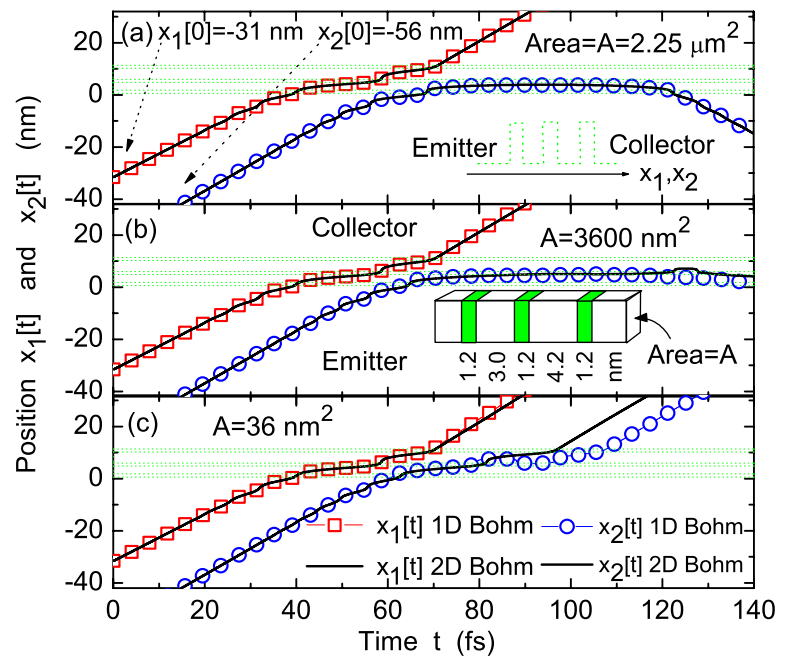

FIG. 1 (color online). Two-particle Bohm trajectories with Coulomb interaction in a triple barrier tunneling scenario computed from our 1D approach (symbols) and from exact 2D results (solid lines) for three different lateral areas that modifies the Coulomb interaction.

second is reflected. However, in Fig. 1(c) the smaller lateral area provides a strong Coulomb interaction between the electrons and the second is finally transmitted because of the "presence" of the first one in the barrier region.

Second algorithm. - We generalize the first algorithm to arbitrary systems with Coulomb and exchange interactions. Since the symmetry of the many-particle wave function is a constant of motion, we introduce the exchange interaction into the initial wave function. We define $\boldsymbol{\Phi}\left(\vec{x}, t_{o}\right)$ as a Slater determinant:

$$
\boldsymbol{\Phi}\left(\vec{x}, t_{o}\right)=C \sum_{l=1}^{N !} \chi_{p(l)_{1}}\left(x_{1}, t_{o}\right) \cdots \chi_{p(l)_{N}}\left(x_{N}, t_{o}\right) s(\vec{p}(l)),
$$

$C$ being a normalization constant. The sum is over all $\mathbf{N}$ ! permutations $\vec{p}(l)=\left\{p(l)_{1}, p(l)_{2}, \ldots, p(l)_{N}\right\}$ and $s(\vec{p}(l))=$ \pm 1 is the sign of the permutations.

Because of the Pauli exclusion principle, the modulus of the wave function tends to zero, $R\left(x_{a}, \vec{x}_{b}[t], t\right) \rightarrow 0$, every position where $x_{a} \rightarrow x_{k}[t]$. Thus, the term $G_{a}\left(x_{a}, \vec{x}_{b}[t], t\right)$ has asymptotes at $x_{a} \rightarrow x_{k}[t]$. Therefore, when the exchange interaction is present, the simplification done in the first algorithm, $G_{a}\left(x_{a}, \vec{x}_{b}[t], t\right) \approx G_{a}\left(x_{a}[t], \vec{x}_{b}[t], t\right)$, is inaccurate. However, by the linearity of Eq. (1), the many-particle wave function subjected to the initial condition (7) can be written everywhere as $\boldsymbol{\Phi}(\vec{x}, t)=$ $C \sum_{l=1}^{N !} \boldsymbol{\Phi}_{\vec{p}(l)}(\vec{x}, t) s(\vec{p}(l))$ where each wave function $\boldsymbol{\Phi}_{\vec{p}(l)}(\vec{x}, t)$ has no exchange symmetry and it can be computed following the first algorithm. Therefore, for each summand and each $x_{a}$ electron, we can compute $\boldsymbol{\Phi}_{\vec{p}(l)}\left(x_{a}, \vec{x}_{b}[t], t\right) \approx \tilde{\boldsymbol{\Psi}}_{a, \vec{p}(l)}\left(x_{a}, t\right) \exp \left[z_{a, \vec{p}(l)}(t)\right] \quad$ with $z_{a, \vec{p}(l)}(t)=\alpha_{a, \vec{p}(l)}(t)-i \beta_{a, \vec{p}(l)}(t)$. The wave function $\tilde{\boldsymbol{\Psi}}_{a, \vec{p}(l)}\left(x_{a}, t\right)$ is the solution of Eq. (6a) with $u_{a}\left(x_{a}, t\right)=$ $U_{a}\left(x_{a}, \vec{x}_{b}[t], t\right)$ when the initial boundary condition is

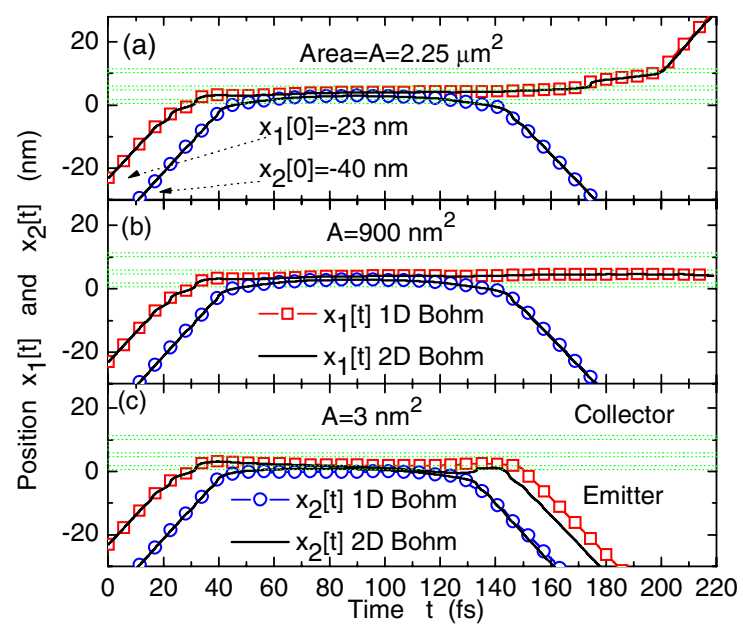

FIG. 2 (color online). Two-particle Bohm trajectories with Coulomb and exchange interactions computed from our 1D approach (symbols) and from 2D exact results (solid lines) for three lateral areas.

$\tilde{\boldsymbol{\Psi}}_{a, \vec{p}(l)}\left(x_{a}, t_{o}\right)=\chi_{p(l)_{a}}\left(x_{a}, t_{o}\right)$. Therefore, the manyparticle wave function at $\left\{x_{a}, \vec{x}_{b}[t]\right\}$ is $\boldsymbol{\Phi}\left(x_{a}, \vec{x}_{b}[t], t\right) \approx$ $C \sum_{l=1}^{N !} \tilde{\mathbf{\Psi}}_{a, \vec{p}(l)}\left(x_{a}, t\right) \exp \left[z_{a, \vec{p}(l)}(t)\right] s(\vec{p}(l))$. Here, the phases $z_{a, \vec{p}(l)}(t)$ are relevant. The permutations $\vec{p}(l)$ and $\vec{p}\left(l^{\prime}\right)$, with $p(l)_{a}=p\left(l^{\prime}\right)_{a}$, leads to an identical wave function $\tilde{\boldsymbol{\Psi}}_{a, \vec{p}(l)}\left(x_{a}, t\right)=\tilde{\boldsymbol{\Psi}}_{a, \vec{p}\left(l^{\prime}\right)}\left(x_{a}, t\right) \equiv \tilde{\boldsymbol{\Psi}}_{a, j}\left(x_{a}, t\right), \quad$ where $j \equiv$ $p(l)_{a}=p\left(l^{\prime}\right)_{a}$. Therefore, we can write $\boldsymbol{\Phi}\left(x_{a}, \vec{x}_{b}[t], t\right) \approx$ $C^{\prime} \sum_{j=1}^{N} \tilde{\boldsymbol{\Psi}}_{a, j}\left(x_{a}, t\right) w_{a, j}(t)$ where we have merged the $N$ ! unknowns, $\exp \left[z_{a, \vec{p}(l)}(t)\right]$, and $s(\vec{p}(l))$ into only $N$ values $w_{a, j}(t)$. In fact, since a global time-dependent constant on the many-particle wave function has no effect on the Bohm velocity (4), only $N-1$ values of $w_{a, j}(t)$ are needed. Because of the Pauli exclusion (i.e., $\boldsymbol{\Phi}\left(x_{a}, \vec{x}_{b}[t], t\right)=0$ for $x_{a}=x_{k}[t]$ with $k \neq a$ ), we obtain $N-1$ conditions for finding $w_{a, j}(t)$, i.e., $\sum_{j=1}^{N} \tilde{\boldsymbol{\Psi}}_{a, j}\left(x_{k}[t], t\right) w_{a, j}(t)=0$ for $k \neq a$. A straightforward solution of the previous system of $N-1$ equations leads to the wave function:

$$
\begin{aligned}
\tilde{\boldsymbol{\Psi}}_{a}\left(x_{a}, t\right)= & \sum_{j=1}^{N !} \tilde{\boldsymbol{\Psi}}_{a, p(j)_{1}}\left(x_{1}[t], t\right) \cdots \tilde{\boldsymbol{\Psi}}_{a, p(j)_{N}}\left(x_{N}[t], t\right) \\
& \times s(\vec{p}(j)) .
\end{aligned}
$$

Thus, the computation of $\vec{x}[t]=\left\{x_{1}[t], \ldots, x_{N}[t]\right\}$ will require finding $N^{2}$ different single-particle wave functions, simultaneously, using (6). Figure 2 shows the excellent agreement between the exact Bohm trajectories and those computed from this second algorithm using the same initial Gaussian wave packets of Fig. 1.

In order to clarify the meaning of indistinguishable particles in the Bohmian language, in Fig. 3 we simulate Bohm trajectories with two particular initial positions and with interchanged initial positions (primes) for the two electrons of Fig. 2(b). By the own construction of the wave function (8), $x_{1}^{\prime}{ }_{1}[t]=x_{2}[t]$ and $x_{2}^{\prime}[t]=x_{1}[t]$. 


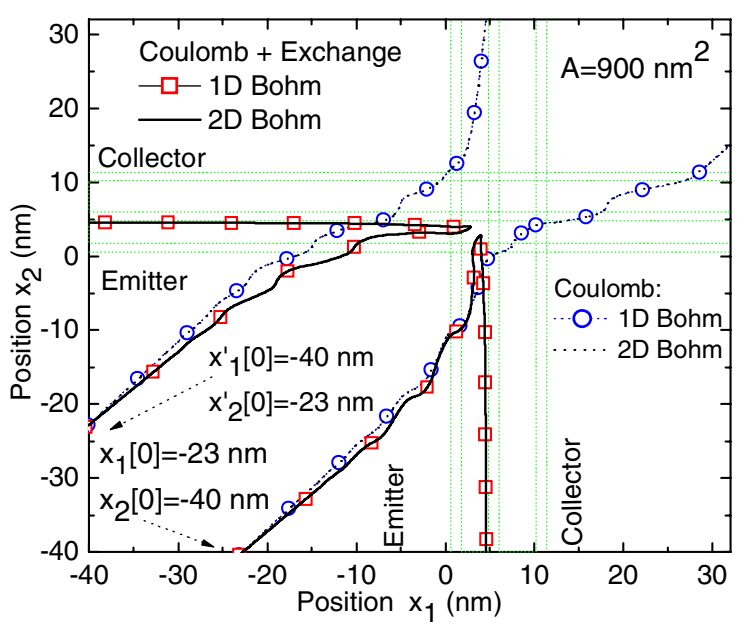

FIG. 3 (color online). Four realizations of two-particle Bohm trajectories interchanging the initial positions with (squares) or without (circles) exchange interaction.

Therefore, although we can distinguish each electron by its own trajectories, one cannot discern between $\left\{x_{1}[t], x_{2}[t]\right\}$ and $\left\{x_{1}^{\prime}{ }_{1}[t], x_{2}^{\prime}{ }_{2}[t]\right\}$ in the observable results. For example, the number of events where $x_{1}[t]$ is transmitted and $x_{2}[t]$ is reflected is exactly identical to the events where $x_{2}[t]$ is transmitted while $x_{1}[t]$ reflected. As seen in Fig. 3, this symmetry is broken without exchange interaction.

For $N>2$, we can estimate the accuracy of our first algorithm by computing $d G_{a}\left(x_{a}, \vec{x}_{b}[t], t\right) / d x_{a}$ at $x_{a}=x_{a}[t]$ for $N-1$ two-electron systems, $\left\{x_{1}, x_{2}\right\}, \ldots,\left\{x_{1}, x_{N}\right\}$, and adding the values. Let us recall that the second algorithm generalizes the first one without any additional approximation but computing $N^{2}$ wave functions. In Fig. 4, we see that the error increases for very small lateral areas (i.e., very strong interactions). This explains the minor divergences in Figs. 1(c) and 2(c). On the contrary, due to the

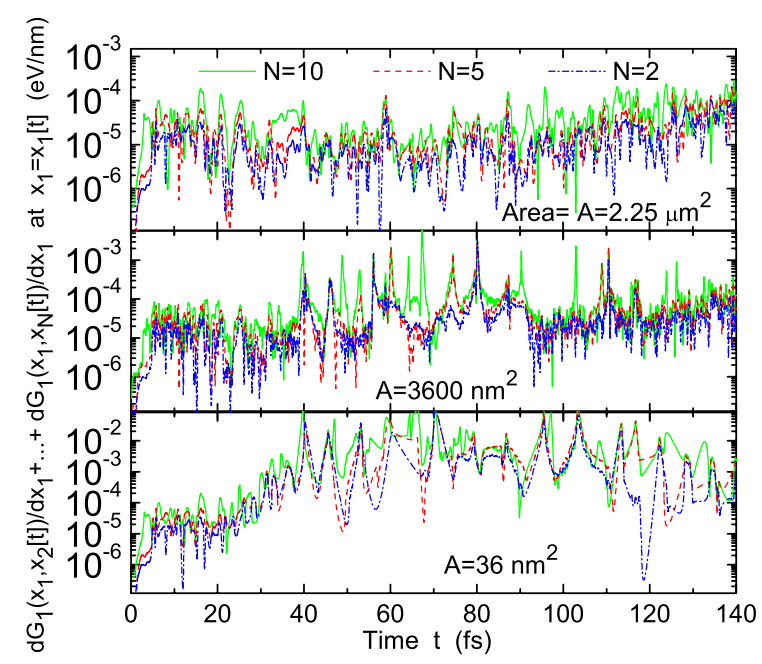

FIG. 4 (color online). Estimation of the error of the first algorithm for different number $N$ of electrons (with arbitrary initial conditions) and three different areas. random nature of $d G_{a}\left(x_{a}, \vec{x}_{b}[t], t\right) / d x_{a}$ (i.e., the correlations are already considered in the algorithms), the error remains roughly equal when $N$ increases.

The work presented here shows the path for a powerful and versatile many-particle quantum transport (Monte Carlo) formalism. The quantum fluctuations of the current can be directly computed, without additional cost, because Bohm trajectories describe the transmission and reflection process as two mutually exclusive events [11]. Since the algorithm deals with time-dependent Schrödinger equations, the many-electron transmission probabilities can be computed for zero or high frequencies, under static or time-dependent external bias [12].

This work was supported through Spanish MEC Project No. TEC2006-13731-C02-02/MIC.

*Electronic address: Xavier.Oriols@uab.es

[1] R. Landauer, Philos. Mag. 21, 863 (1970); S. Datta, Superlattices Microstruct. 28, 253 (2000); W. R. Frensley, Rev. Mod. Phys. 62, 745 (1990); M. Buttiker, Phys. Rev. Lett. 65, 2901 (1990).

[2] W. Kohn and L. J. Sham, Phys. Rev. 140, A1133 (1965).

[3] M. Brandbyge, J. L. Mozos, P. Ordejon, J. Taylor, and K. Stokbro, Phys. Rev. B 65, 165401 (2002); S. Kurth, G. Stefanucci, C. O. Almbladh, A. Rubio, and E. K. U. Gross, Phys. Rev. B 72, 035308 (2005).

[4] D. Bohm, Phys. Rev. 85, 166 (1952).

[5] P. R. Holland, The Quantum Theory of Motion (Cambridge University Press, Cambridge, England, 1993).

[6] B. G. Englert, M. O. Scully, G. Sussmann, and H. Walther, Z. Naturforsch. 47, 1175 (1992); D. Dürr, W. Fusseder, S. Goldstein, and N. Zanghi, Z. Naturforsch. 48, 1261 (1993); C. Dewdney, L. Hardy, and E. J. Squires, Phys. Lett. A 184, 6 (1993).

[7] D. K. Ferry and J.-R. Zhou, Phys. Rev. B 48, 7944 (1993); C. L. Lopreore and R. E. Wyatt, Phys. Rev. Lett. 82, 5190 (1999); A. Donoso and C. C. Martens, Phys. Rev. Lett. 87, 223202 (2001); B. K. Dey, A. Askar, and H. Rabitz, J. Chem. Phys. 109, 8770 (1998).

[8] X. Oriols, Phys. Rev. A 71, 017801 (2005).

[9] The computation of a $N=2$ trajectory in a dual xenon $2 \times 3.06 \mathrm{GHz}$ server takes $\sim 20 \mathrm{~h}$ when solving Eq. (1), whereas it takes $\sim 50 / 100 \mathrm{~s}$ using our algorithms.

[10] In the numerical examples, the potential energy $U\left(\vec{r}_{1}, \vec{r}_{2}, t\right)$ is computed from the numerical solution of the $3 \mathrm{D}$ Poisson equation in the $3 \mathrm{D}$ volume seen in the inset of Fig. 1(b) with the following boundary conditions: on the emitter surface, $0 \mathrm{~V}$; on the collector surface, $0.05 \mathrm{~V}$; and an electric field equal to zero (Neumann conditions) on the rest of the surfaces. The $3 \mathrm{D}$ potential is averaged over the lateral surfaces $\left\{y_{1}, z_{1}\right\}$ and $\left\{y_{2}, z_{2}\right\}$ to obtain $U\left(x_{1}, x_{2}, t\right)$ in (1). On the other hand, the potential energy for $x_{1}$ electron in (6) is obtained by fixing $x_{2}=x_{2}[t]$, i.e., $u_{1}\left(x_{1}, t\right)=$ $U\left(x_{1}, x_{2}[t], t\right)$. Equivalently, $u_{2}\left(x_{2}, t\right)=U\left(x_{1}[t], x_{2}, t\right)$.

[11] X. Oriols, A. Trois, and G. Blouin, Appl. Phys. Lett. 85, 3596 (2004).

[12] X. Oriols, A. Alarcón, and E. Fernàndez-Díaz, Phys. Rev. B 71, 245322 (2005). 